\title{
The Dust around Cool Stars
}

\section{IRAKLI SIMONIA and TSITSINO SIMONIA}

\section{Tbilisi Laboratory of the Abastumani Astrophysical Observatory Tbilisi, Republic of Georgia}

The present work deals with some properties of cool, crystalline hydrocarbons in the dust shells around stars of the late types, studied on the basis of laboratory investigations of terrestrial crystalline hydrocarbons. It is common knowledge that solid particles of silicon carbide, graphite, etc., can form in the vicinity of carbon stars. Taking into account the chemical composition of carbon stars and the complex structure of the "star - dust shell" system, one can suggest that the dust around stars of the types mentioned may contain solid carbon particles formed as separate equally-dispersed crystals, as well as complex, unequally dispersed polycrystals. One of the most interesting properties of solid crystalline hydrocarbons is their ability to luminesce when excited by UV radiation. Terrestrial crystalline hydrocarbons of the aromatic series obtained from different grades of petroleum show luminescence only under UV radiation of 3600-3800 $\AA$. Luminescence of such hydrocarbons occurs in the yellow-green part of the visible spectrum, with a maximum wavelength of $5500 \AA$. When the intrinsic temperature of the hydrocarbons is $270 \mathrm{~K}$ and higher, the luminescence of terrestrial hydrocarbons is of the fluorescence type. However, at intrinsic temperatures below $270 \mathrm{~K}$, the luminescence is of the phosphorescence type. When hydrocarbons are heated up to $310 \mathrm{~K}$, they appear to lose their luminescent properties. In the outer layers of detached dust shells surrounding cool stars, crystalline hydrocarbons can be heated to moderate temperatures. If those slightly heated crystalline hydrocarbons happen to receive UV radiation incident from a distant foreign source, those hydrocarbons will luminesce in the corresponding spectrum. This effect can be termed "weak episodic phosphorescence." It may be observed as a blue or violet glow. The results of these laboratory studies of terrestrial crystalline hydrocarbons can be applied in investigations of other physical and chemical properties of crystalline hydrocarbons found in the dust shells of cool stars. 